\title{
The Galactic Center region imaged with MAGIC and variability searches during the G2 pericenter passage
}

\section{Christian Fruck $^{* a}$, John E. Ward ${ }^{b},{\text { levgen } \operatorname{Vovk}^{a}, \text { Marcel Strzys }^{a} \text { and Gaia Vanzo }}^{c}$ for the MAGIC collaboration}

${ }^{a}$ Max-Planck-Institut für Physik, D-80805 München, Germany

${ }^{b}$ Institut de Fisica d'Altes Energies, Edifici Cn., E-08193 Bellaterra (Barcelona), Spain

${ }^{c}$ Università di Padova and INFN, 35131, Padova, Italy

E-mail: fruckempp.mpg.de, jwardeifae.es

The Major Atmospheric Gamma Imaging Cherenkov (MAGIC) telescopes have observed the Galactic Center during the past four years. Due to its location in the northern hemisphere, MAGIC observes the GC at large zenith angles (58-70 deg), resulting in a higher energy threshold, but an enhanced effective collection area at multi-TeV energies.

The primary goal of this campaign was searching for variability in the very high energy gamma ray regime during the closest approach of the G2 gas cloud, which orbits the GC on a highly eccentric trajectory with a pericentre distance of only a few thousand Schwarzschild radii. These observations, apart from searching for variability, allowed us to perform morphological and spectral studies of the region, based on a large data set of about $80 \mathrm{~h}$.

No variability was detected in the 2013 and the 2014 data set. The diffuse TeV emission around GC could be imaged with great sensitivity, which led to the detection of $\mathrm{TeV}$ gamma rays from a region 0.2 deg away from SgrA* that is spatially coincident to the extended radio structure called Arc.

The 34th International Cosmic Ray Conference,

30 July- 6 August, 2015

The Hague, The Netherlands

\footnotetext{
* Speaker.
} 


\section{Introduction}

\subsection{The Galactic Center Region}

The central region of our galaxy is very densely populated with a large variety of astrophysical objects, many of which may be sites of extreme particle acceleration and hence gamma-ray emission. The most particular candidate for such an accelerator is the super massive black hole $(\mathrm{SMBH})$, which is identified with the point-like radio source SgrA* and known to be the dynamical center of stellar motion in the central region of our galaxy [1, 2, 3, 4].

The Galactic center (GC) has also been extensively observed in the high energy (HE; $\gtrsim 100$ $\mathrm{MeV}$ ) regime with the EGRET and Fermi-LAT instruments and in the very high energy (VHE, $\gtrsim 100 \mathrm{GeV}$ ) regime with Imaging Atmospheric Cherenkov Telescopes [5, 6, 7, 8, 9]. More recent observations have shown that part of the $\mathrm{TeV}$ emission comes from a point-like object that coincides with the location of $\mathrm{SgrA}^{*}$ within angular resolution and revealed that the area hosts an extended emission region, the $\mathrm{GC}$ ridge $[10,11,12]$.

\subsection{The periastron passage of the G2 Gas Cloud}

The G2 gas cloud was discovered in VLT data and found to be on a highly eccentric orbit towards the central black hole (BH) of our galaxy [13]. This object with an estimated mass of about three times the Earth's was predicted to experience its periastron passage around mid 2013 at a distance of about 2200 Schwarzschild radii [14].

It is still subject to discussion, weather G2 is actually a gas cloud or rather a star or a star with stellar wind $[15,16]$. Predictions vary from no effect at all to strong flaring activity of SgrA* $[17,18]$. Some suggest that G2 may interact with stellar mass black holes that are expected to exist in the vicinity of SgrA* [19].

Regardless of the poor knowledge of the exact properties of G2, given the rarity of such an event, instruments in many wavebands started monitoring campaigns of SgrA*. So far no detections of abnormal activity have been reported [20, 21, 22]. There is however a hint for increased flaring activity in X-ray during 2013/14 [23].

\subsection{The MAGIC Telescopes}

The MAGIC (Major Atmospheric Gamma Imaging Cherenkov) telescopes are two $17 \mathrm{~m}$ diameter Imaging Atmospheric Cherenkov Telescopes (IACT). They are located at a height of 2200 $\mathrm{m}$ a.s.l. in the Roque de los Muchachos Observatory on the Canary Island of La Palma, Spain $\left(28^{\circ} \mathrm{N}, 18^{\circ} \mathrm{W}\right)$. The telescopes are used for observations of Cherenkov light flashes produced by superluminal charged particles in Extensive Air Showers (EAS) initiated by gamma-ray photons with energies $\gtrsim 50 \mathrm{GeV}$.

Both telescopes are nominally operated together in the so-called stereoscopic mode, in which only events seen simultaneously in both telescopes are triggered and analyzed [24]. At energies around a few hundred $\mathrm{GeV}$, for low Zenith distance ( $\mathrm{Zd}$ ) observations, the energy resolution is approximately $16 \%$ with a systematic bias on the order of $15 \%$ [25], while the angular resolution of the system is $\lesssim 0.07^{\circ}$. The sensitivity above $220 \mathrm{GeV}$ is $(0.66 \pm 0.03) \%$ Crab Units (CU) for 50 hours of observations at low zenith angles. 


\subsection{The MAGIC GC Observation Campaign}

The GC region observing campaign began in April 2012, and was intended as a long-term monitoring program spanning over several years. Since then around 80 hours of good quality data have been recorded.

When observed from the MAGIC site, the GC culminates at $57.78^{\circ} \mathrm{Zd}$. This has strong implications for the observation conditions and the available time slots for observations. When observing at such large inclination $\left(58^{\circ}-70^{\circ}\right)$ the Cherenkov light pool at ground level produced by EAS is increased by a factor 4 to 10 in area. This implies a higher energy threshold, but also leads to an increased collection area by around this factor. Such observations with the MAGIC telescopes provide excellent sensitivity for energies $\gtrsim 1 \mathrm{TeV}$. The time window for observing the GC with MAGIC when setting the $\mathrm{Zd}$ limit to $70^{\circ}$ is approximately from mid-February until the end of September.

\section{Data Analysis and Results}

\subsection{Data selection}

Given that the GC was observed at such large Zd, the impact of the atmosphere on the data quality is increased compared to low $\mathrm{Zd}$ observations. This accounts for the effect of decreased transmission, as well as the increase of scattered light from the moon or terrestrial sources. The measurement of flux above a certain energy threshold is most sensitive to changes of atmospheric transmission. Therefore two different data quality selection classes were introduced: one for spectral and morphological studies and one with even stricter selection criteria for producing the light curve.

\subsection{Data Analysis}

After applying quality cuts the data could be analyzed with the standard MAGIC software tools starting from single telescope image parameter files, that are then combined through the calculation of stereo parameters.

Random Forests (RFs) based on simulated gamma rays are used for event classification, direction reconstruction and gamma-hadron separation. All events classified as gamma rays are used for the extraction of signal and background from suitable regions. SgrA* was observed in the socalled Wobble mode, where the source is observed in symmetric positions in the camera plane, all with an offset of $0.4^{\circ}$ from the camera center. For the production of the spectrum and light curve, symmetric regions that are located off the Galactic plane were used for the background estimation. For the production of sky maps the blind map method was used, which compares the camera positions exposed to different regions of the sky for finding the best background estimate for the given camera position.

\subsection{Variability Search}

For the production of the light curve only data that fulfilled selection criteria for very clear skies was used, which is currently available only for the 2013 and 2014 data. The total amount of data in this sample corresponds to an observation time of about $45 \mathrm{~h}$. The flux from a region of 


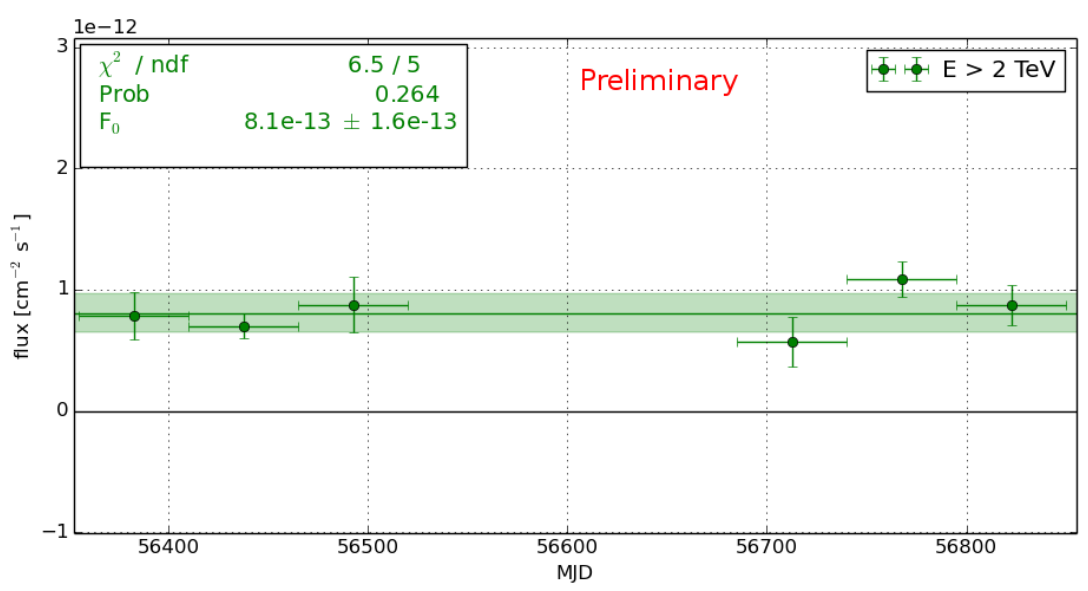

Figure 1: Light curve of integral flux $F$ above $2 \mathrm{TeV}$ from $0.14^{\circ}$ radius around SgrA* with 50d binning for 2013 and 2014 data

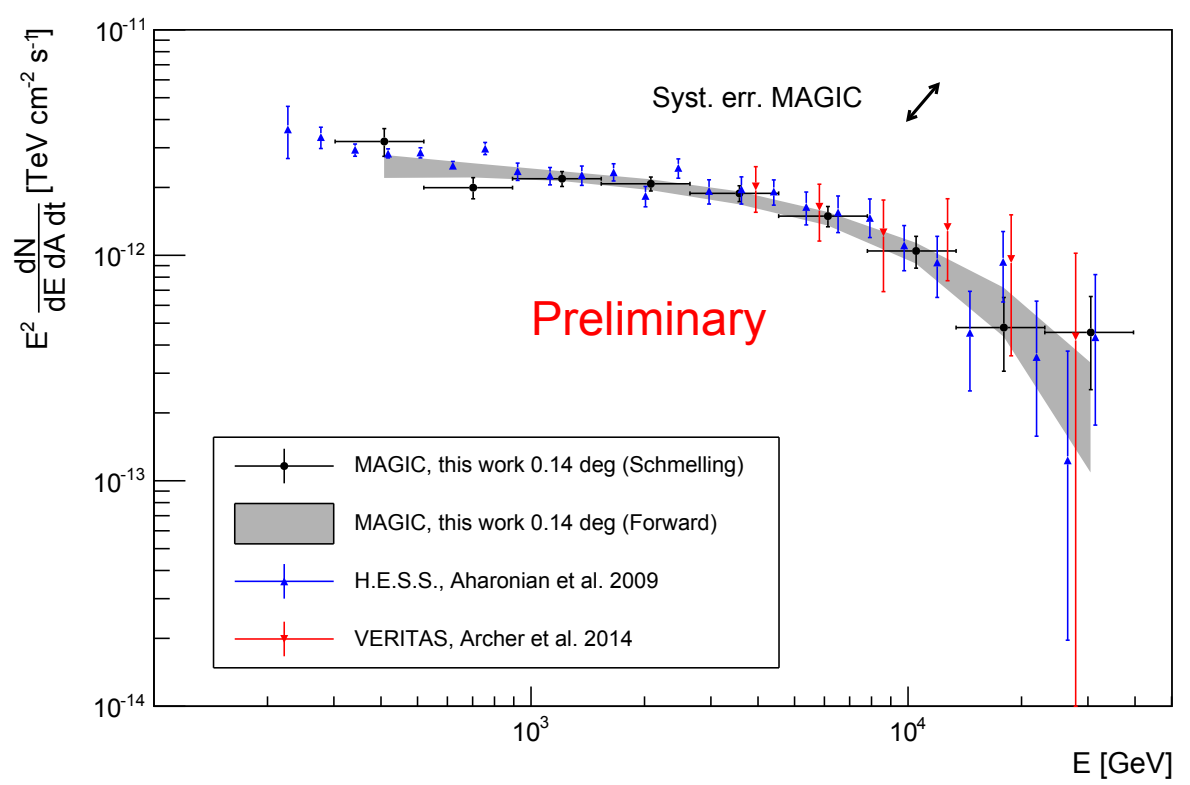

Figure 2: SED of the GC point-source extracted from a $0.14^{\circ}$ radius region around $\mathrm{SgrA}^{*}$, compared to previous measurements by H.E.S.S. and VERITAS. MAGIC data is shown as unfolded spectrum (data points) and power-law function with cut-off from froward folding (shaded region).

radius $0.14^{\circ}$ around the location of $\mathrm{SgrA}^{*}\left(\mathrm{RA} 266.4168^{\circ}\right.$, Dec $-29.0078^{\circ}$ ) was measured in time bins of 50d length. Figure 1 shows the light curve of the integral flux $F$ above $2 \mathrm{TeV}$ for 2013 and 2014 data. No significant deviation from a constant flux has been found.

\subsection{Spectrum of the SgrA* point source}

The same extraction region that was used for the light curve was also used for the SED, but using the data set with slightly relaxed data selection criteria and for the years 2012, 2013, 2014 and 2015 (in total $\sim 80$ h). Figure 2 shows the SED obtained from the new observations by MAGIC compared to previous measurements by H.E.S.S. and VERITAS [10, 12]. The best fitting parame- 


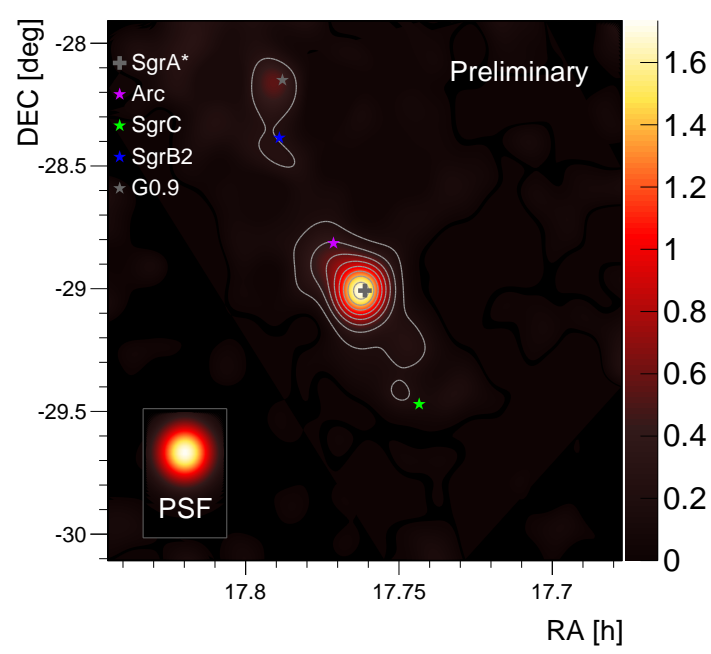

(a)

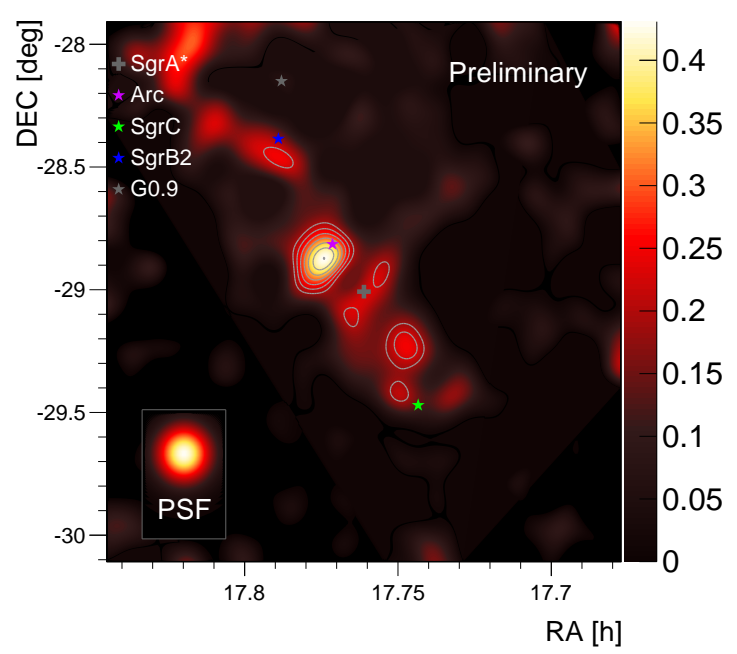

(c)

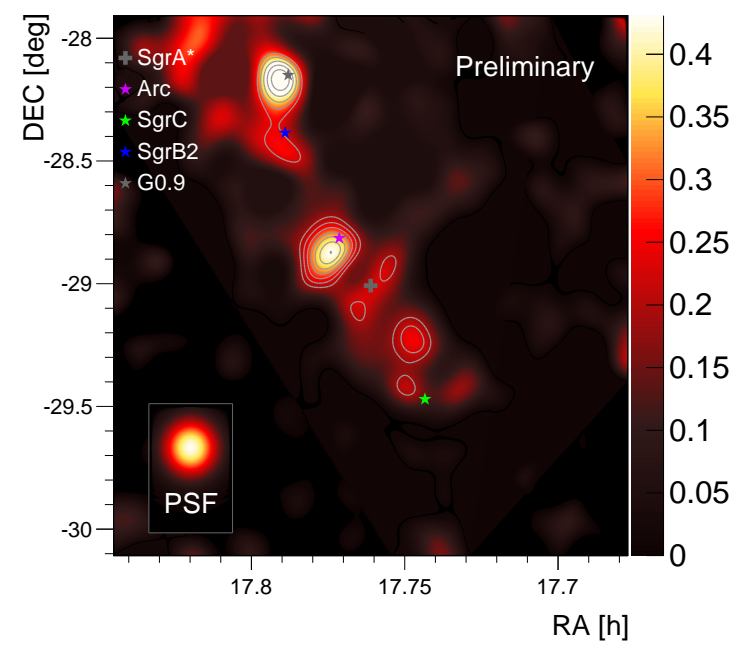

(b)

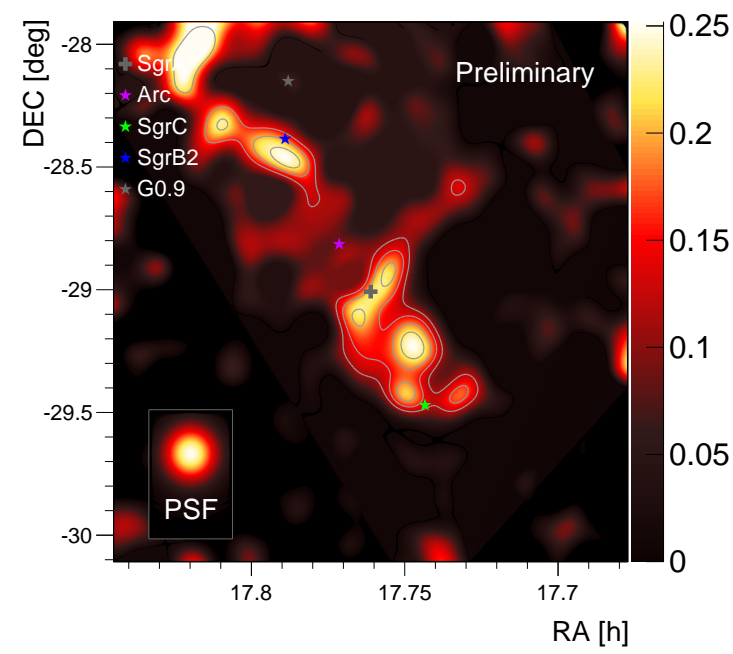

(d)

Figure 3: Sky maps based on $80 \mathrm{~h}$ data set for energy range of $E \gtrsim 1 \mathrm{TeV}$ : relative flux map with significance contours starting from a minimum of $5 \sigma$ local significance (a), residual map after subtracting the fitted SgrA* point source (b), after subtracting G0.9+0.1 in addition (c), residual map after subtracting also the emission region close to the arc with significance contours starting at $3 \sigma(d)$.

ters when assuming a power-law function with exponential cut-off

$$
\frac{\mathrm{d} F}{\mathrm{~d} E}=f_{0}\left(\frac{E}{\mathrm{TeV} 1}\right)^{\alpha} \exp -\frac{E}{E_{\mathrm{cut}}} .
$$

and using the forward folding method are $\alpha=2.04 \pm 0.12$ and $E_{\text {cut }}=13.5 \pm 5.9 \mathrm{TeV}$.

\subsection{Morphology Study}

The sky maps that are discussed in the following are based on the same 80h data set that was used for the SED and use an energy range of $E \gtrsim 1 \mathrm{TeV}$. Apart from the SgrA* point-source, like in 
previous measurements by H.E.S.S. and VERITAS [26, 12], the MAGIC measurements also show extended emission coming from the region along the Galactic plane. This becomes more obvious when fitting the emission within $0.12^{\circ}$ from SgrA* source with a $2 \mathrm{D}$ Gaussian and subtracting it from the relative flux map (see figure $3 \mathrm{a}$ ). The residual sky map shows significant gamma-ray excess from the location of the supernova remnant G0.9+0.1 as well as from a region $\sim 0.2^{\circ}$ left from $\operatorname{SgrA}^{*}$ (figure 3b).

G0.9+0.1 has been previously detected in VHE gamma rays by the H.E.S.S telescope [27] and has been identified as VHE counterpart of the PWN. The second region with strong gamma-ray excess, which has not been reported in the literature to date, is seen with a local significance of more than $7 \sigma$ when subtracting the contribution from $\operatorname{SgrA}^{*}$ as specified above (see figure $\left.3 \mathrm{~b}\right)^{1}$. This location coincides with the southern part of the large scale radio structure called "the Arc" and hence it may be physically associated with it [28]. Other possible counterparts are the super shell G0.11-0.11, also possibly interacting with the Arc [29], or the pulsar wind nebula (PWN) G0.130.11 [30]. It is also possible that the $\mathrm{TeV}$ emission is produced by the interaction of cosmic rays (e.g. accelerated in a flaring period of SgrA* in the recent past) with the dense molecular clouds in this region [31].

After fitting and removing also those two sources in a similar manner as described above, the residual flux distributes along the Galactic plane (extending from top left to bottom right of the sky map), with evidence for enhanced emission from the dense molecular clouds SgrB2 and SgrC as well as from the region of $\operatorname{SgrD}$ (figure 3d).

\section{Conclusions}

The Galactic Center has been re-observed by MAGIC during 2012-15, accumulating approximately $80 \mathrm{~h}$ of good quality data. These observations were carried out in order to probe for flux variability of the $\mathrm{SgrA}^{*} \mathrm{TeV}$ source during the periastron passage of the $\mathrm{G} 2$ gas cloud.

No variability has been detected during the years 2013/14. Apart from probing for variability, the data could also be used for a precise spectral and morphological analysis of the SgrA* point-source and its surroundings. The flux and spectral shape has been found in good agreement with measurements by other instruments. In addition, we found a significant VHE gamma-ray excess from a region 0.2 deg away from $\mathrm{SgrA}^{*}$ and spatially coinciding with the Arc radio source. The significance and morphology of this potential new source will be further investigated under different assumptions for the diffuse VHE gamma-ray backgrounds in that region of the sky.

\section{Acknowledgments}

We would like to thank the Instituto de Astrofisica de Canarias for the excellent working conditions at the Observatorio del Roque de los Muchachos in La Palma. The financial support of the German BMBF and MPG, the Italian INFN and INAF, the Swiss National Fund SNF, the ERDF under the Spanish MINECO (FPA2012-39502), and the Japanese JSPS and MEXT is gratefully acknowledged. This work was also supported by the Centro de Excelencia Severo Ochoa

\footnotetext{
${ }^{1}$ A different parametrization of the diffuse background contribution may lead to different results.
} 
SEV-2012-0234, CPAN CSD2007-00042, and MultiDark CSD2009-00064 projects of the Spanish Consolider-Ingenio 2010 programme, by grant 268740 of the Academy of Finland, by the Croatian Science Foundation (HrZZ) Project 09/176 and the University of Rijeka Project 13.12.1.3.02, by the DFG Collaborative Research Centers SFB823/C4 and SFB876/C3, and by the Polish MNiSzW grant 745/N-HESS-MAGIC/2010/0.

\section{References}

[1] R. Davies, D. Walsh, and R. Booth, The radio source at the galactic nucleus, Monthly Notices of the Royal Astronomical Society 177 (1976), no. 2 319-333.

[2] R. Ekers et al., The radio structure of sgr a, Astronomy and Astrophysics 122 (1983) 143-150.

[3] R. Genzel and A. Eckart, The galactic center black hole, in The Central Parsecs of the Galaxy, vol. 186, p. 3, 1999.

[4] R. Schödel et al., A star in a 15.2-year orbit around the supermassive black hole at the centre of the milky way, Nature 419 (2002), no. 6908 694-696.

[5] R. Enomoto, M. Mori, and S. Yanagita, The Universe Viewed in Gamma-rays: International Science Symposium on the Universe Viewed in Gamma-Rays, Held on September 25 - 28, 2002, in Kashiwa, Chiba, Japan. Frontiers science series. Universal Academy Press, 2003.

[6] K. Kosack et al., Tev gamma-ray observations of the galactic center, The Astrophysical Journal Letters 608 (2004), no. 2 L97.

[7] K. Tsuchiya et al., Detection of sub-tev gamma rays from the galactic center direction by cangaroo-ii, The Astrophysical Journal Letters 606 (2004), no. 2 L115.

[8] F. Aharonian et al., Very high energy gamma rays from the direction of sagittarius a*., Astronomy \& astrophysics. 425 (2004), no. 1 L13-L17.

[9] J. Albert et al., Observation of gamma rays from the galactic center with the magic telescope, The Astrophysical Journal Letters 638 (2006), no. 2 L101.

[10] F. Aharonian et al., Spectrum and variability of the galactic center vhe $\gamma$-ray source hess j1745-290, Astronomy \& Astrophysics $\mathbf{5 0 3}$ (2009), no. 3 817-825.

[11] F. Acero et al., Localizing the vhe $\gamma$-ray source at the galactic centre, Monthly Notices of the Royal Astronomical Society 402 (2010), no. 3 1877-1882.

[12] A. Archer et al., Very-high energy observations of the galactic center region by veritas in 2010-2012, The Astrophysical Journal 790 (2014), no. 2149.

[13] S. Gillessen et al., A gas cloud on its way towards the supermassive black hole at the galactic centre, Nature 481 (2012), no. 7379 51-54.

[14] S. Gillessen et al., New observations of the gas cloud g2 in the galactic center, The Astrophysical Journal 763 (2013), no. 278.

[15] K. Phifer et al., Keck observations of the galactic center source g2: Gas cloud or star?, The Astrophysical Journal Letters 773 (2013), no. 1 L13.

[16] N. Scoville and A. Burkert, The galactic center cloud g2? a young low-mass star with a stellar wind, The Astrophysical Journal 768 (2013), no. 2108. 
[17] M. Schartmann et al., Simulations of the origin and fate of the galactic center cloud g2, The Astrophysical Journal 755 (2012), no. 2155.

[18] D. Giannios and L. Sironi, The 2 star as a probe of the accretion disc of sgr $a^{*}$, Monthly Notices of the Royal Astronomical Society: Letters 433 (2013), no. 1 L25-L29.

[19] I. Bartos et al., Gas cloud g2 can illuminate the black hole population near the galactic center, Physical review letters 110 (2013), no. 22221102.

[20] M. Valencia-S et al., Monitoring the dusty s-cluster object (dso/g2) on its orbit toward the galactic center black hole, The Astrophysical Journal 800 (2015), no. 2125.

[21] M. Tsuboi et al., No microwave flare of sagittarius $a^{*}$ around the $g 2$ periastron passing, The Astrophysical Journal Letters 798 (2015), no. 1 L6.

[22] G. C. Bower et al., Radio and millimeter monitoring of sgr a*: Spectrum, variability, and constraints on the g2 encounter, The Astrophysical Journal 802 (2015), no. 169.

[23] G. Ponti et al., Fifteen years of xmm-newton and chandra monitoring of sgr a*: Evidence for a recent increase in the bright flaring rate, arXiv preprint arXiv:1507.02690 (2015).

[24] J. A. others, The major upgrade of the MAGIC telescopes, part ii: A performance study using observations of the crab nebula, Astroparticle Physics (2015), no. 0 -.

[25] J. A. others, Performance of the $\{M A G I C\}$ stereo system obtained with crab nebula data, Astroparticle Physics 35 (2012), no. 7435 - 448.

[26] F. Aharonian et al., Discovery of very-high-energy $\gamma$-rays from the galactic centre ridge, Nature 439 (2006), no. 7077 695-698.

[27] H. Collaboration and F. Aharonian, Very high energy gamma rays from the composite snr g0. 9+ 0.1, arXiv preprint astro-ph/0501265 (2005).

[28] C. Fruck, The Galactic Center resolved with MAGIC and a new technique for Atmospheric Calibration. Ph.d. thesis, Technical University Munich, April, 2015.

[29] M. Tsuboi, N. Ukita, and T. Handa, An expanding shell-like molecular cloud near the galactic center arc, The Astrophysical Journal 481 (1997), no. 1263.

[30] Q. D. Wang, F. Lu, and C. C. Lang, X-ray thread g0. 13-0.11: A pulsar wind nebula?, The Astrophysical Journal 581 (2002), no. 21148.

[31] T. Handa et al., Thermal sio and h13co+ line observations of the dense molecular cloud g0. 11-0.11 in the galactic center region, The Astrophysical Journal 636 (2006), no. 1261. 